\title{
ESPACIALIDADES PEREGRINAS: EL CASO DE LA PEREGRINACIÓN JUVENIL A PIE A LUJÁn.
}

- FABiÁn ClAUdio FLORES 1

RESUMEN: DESDE EL AÑO 1975 SE LLEVA A CABO UNA DE LAS PEREGRINACIONES CATÓLICAS MÁS IMPORTANTES DE LA ARGENTINA: LA PEREGRINACIÓN JUVENIL A PIE A LA BASÍLICA DE LUJÁN. BAJO EL LEMA: "LA JUVENTUD PEREGRINA A LUJÁN POR LA PATRIA”, EL 25 DE OCTUBRE DE 1975 UNOS 30 MIL FIELES PEREGRINARON POR PRIMERA VEZ A ESA HIERÓPOLIS ARGENTINA. EL RECORRIDO PREESTABLECIDO CONSTA DE UNOS $60 \mathrm{KM}$. APROXIMADAMENTE DESDE EL BARRIO DE LINIERS EN LA CABA HASTA LA BASÍLICA EN LUJÁN, PROMEDIANDO LAS 15 HORAS DE CAMINATA. NO TODOS LAS REALIZAN COMPLETA SINO QUE SE SUMAN EN LAS DISTINTAS LOCALIDADES DEL TRAYECTO, ESPECIALMENTE ENTRE MORÓN Y MORENO. EN TODO EL RECORRIDO Y LA PRÁCTICA PEREGRINA, LAS ESPACIALIDADES JUEGAN UN ROL CENTRAL EN LAS NARRATIVAS DE LOS DEVOTOS DE UN HETEROGÉNEO ORIGEN. LOS OBJETIVOS DEL TRABAJO SON DOS: POR UN LADO, ANALIZAR UTILIZANDO LA METODOLOGÍA CUALITATIVAS -PROPIA DE LA NUEVA GEOGRAFÍA CULTURAL- EL ORIGEN Y LA TRANSFORMACIÓN DE LA PRÁCTICA PEREGRINA EN GENERAL; Y POR OTRO LADO, EXPLORAR LAS FORMAS QUE ADOPTA ESTA PEREGRINACIÓN EN PARTICULAR "LA DE LOS JÓVENES" EN RELACIÓN CON EL CONTEXTO Y LA OFERTA MÁS AMPLIA DEL CALENDARIO PEREGRINO A LA CIUDAD DE LUJÁN.

PALABRAS CLAVES: PEREGRINACIÓN - ESPACIALIDAD - RELIGIOSIDAD. 
La peregrinación como fenómeno espacial

Dentro de las ciencias sociales de la religión, el tema de la movilidad religiosa ha sido una constante a lo largo de las últimas décadas. Si bien es cierto que la presencia de las motivaciones y los perfiles de los sujetos móviles han ocupado un lugar preponderante en gran parte de los estudios, en los trabajos recientes ha comenzado a advertirse un viraje importante en las maneras de abordar el problema saltando escollos que intentaban proponer miradas clasificatorias que apuntaban con exclusividad a estas dos variables.

Por otro lado, la presencia de estudios referidos al universo católico ocupan un lugar mayoritario, aunque también cabe agregar que se ha ido incrementando el número de experiencias que atraviesan las distintas religiones como el budismo, el hinduismo, el judaísmo y el Islam, entre otros ${ }^{2}$.

La peregrinación como fenómeno espacial es una práctica muy antigua que ha ido sufriendo transformaciones a través del tiempo y de acuerdo a los escenarios donde se ha ido desarrollando, por eso es fundamental el análisis territorial relacional que lo vincule con los contextos sociales, económicos y políticos en torno a los cuales se despliegan estas prácticas socioespaciales.

La amplitud de experiencias del peregrinar y eventos que se vinculan a ellas condujo al acrecentamiento del abanico de posibles líneas de exploración. Así, la categoría de peregrinación se comienza a utilizar para fenómenos más diversos que desbordan lo puramente religioso y se proyectan a situaciones consideradas "seculares". Collins-Kreiner menciona:

"for example, visits to war graves or the graves and residences of celebrities, visits to churchyards and to funerary sites, as sacred and secular pilgrimage. One example is Elvis Presley's mansion and tomb in Memphis. Pilgrims, religious or secular, often share the trait of searching for a meaningful and spiritual experience. These experiences can be described in various ways: transformation, enlightenment, lifechanging events or consciousnesschanging events, but words

seem 
inadequate to describe experiences that often are not amenable to reason" (COLLINSKREINER, 2010: 157).

Desde los años 1990 -entoncesse puede advertir cómo el individuo y su experiencia (personal y social) se han convertido en el centro de interés de los estudios territoriales de las peregrinaciones (SMITH, 1992; COHEN, 1992; FLEISHER, 2000) en un tránsito que ha saltado de estudiarlas desde sus aspectos endógenos hacia una perspectiva más egocéntrica donde se focaliza la experiencia subjetiva de los peregrinos, sus impresiones individuales y las referencias espaciales que hay al respecto (PORIA et. al., 2003, 2004 y 2006).

Entendemos entonces a las peregrinaciones como fenómenos territoriales multidimensionales $\mathrm{y}$ complejos en donde se llevan a cabo una serie de prácticas espaciales a lo largo de un recorrido cuyo final es el destino de esa experiencia sagrada individual, pero que tienen carácter y connotaciones sociales, y que está inmerso en tramas más amplias sociales, económicas, políticas, institucionales y culturales que son fundamentales para entender la espacialidad peregrina.
Numerosos aspectos pueden ser abordados desde lo territorial si situamos la mirada en los componentes simbólicos que surgen del fenómeno: la peregrinación como acto ritual, la imaginación geográfica ${ }^{3}$ y las representaciones socio-territoriales, los sentidos de los itinerarios devocionales y las performatividades peregrinas; este último aspecto es uno de los menos explorados desde los estudios espaciales, vinculando la cuestión del cuerpo-peregrino y las emociones en clave espacial 4 .

Más allá de las diferencias y discusiones en el campo académico, no caben dudas que el fenómeno de la movilidad devocional es una práctica que se ha ido incrementando $\mathrm{y}$ diversificando significativamente en las últimas décadas. El calendario de las rutas peregrinas en la Argentina crece año a año y se suman nuevos destinos, nuevas experiencias y formas que adquiere el viaje religioso, y además- cómo bien resalta Carballo (2010) "la experiencia religiosa peregrina no se restringe solamente a los bienes que ofrece el campo católico institucional, sino que convergen otras necesidades de contacto con lo 
sagrado, donde lo cultural y lo social configuran el espacio" (CARBALLO, 2010: 128).

Hay un constante proceso de reinvención del fenómeno peregrino y las lógicas que lo organizan y le dan sentido.

El modelo de la peregrinación a Santiago de Compostela (tan estudiado desde diferentes aspectos) ya no es útil para entender las peregrinaciones contemporáneas y las prácticas de sacralización de los sujetos en el territorio. De hecho, la historia social del concepto muestra que con el correr del tiempo el término peregrino fue restringiendo su significado para referirse a aquellos sujetos que, inducidos por la fe, marchaban a Santiago de Compostela, mientras que se usaba el de romeros para nombrar a aquellos que tenían a la ciudad de Roma como destino final de su marcha, y como palmeros a los que se dirigían a Jerusalén (PORCAL GONZALO, 2006). Esta diferenciación luego se fue esfumando para aludir en términos genéricos a toda forma de viaje de religioso, independiente del sitio de destino.

Las nuevas lógicas de organización y los contextos contemporáneos dan cuenta de formas pos-modernas del peregrinar que han configurado un sujeto peregrino distinto al tradicional:

$$
\begin{aligned}
& \text { "Diferente do } \\
& \text { peregrino do } \\
& \text { catolicismo } \\
& \text { tradicional, este } \\
& \text { acredita que o sagrado } \\
& \text { já existe dentro dele, } \\
& \text { em seu self, sendo que } \\
& \text { ele precisa estar no } \\
& \text { lugar sagrado para que } \\
& \text { ocorra } \\
& \text { a } \\
& \text { transcendência" } \\
& \text { (OLIVEIRA, 2014: 13). }
\end{aligned}
$$

\section{El peregrino como sujeto espacial}

Las investigaciones geográficas que inauguran el nuevo siglo XXI se posicionan con una fuerte perspectiva cultural que implica un retorno al componente subjetivo, recuperando la centralidad de los actores en el proceso de producción de la espacialidad. El espacio -así concebido- también es la inmaterialidad asociada al componente material; sólo basta para ello como había aclamado el geógrafo británico Edward Soja, "ampliar la imaginación geográfica” (SOJA, 1996) y lograr la inclusión de lo imaginario en geografía que tiene la virtud de desafiar

$$
\begin{gathered}
\text { "la } \\
\text { construcción de }
\end{gathered}
$$


nuevas articulaciones analíticas entre diversas escalas, sujetos, y también entre materialidades $\mathrm{y}$ las inmaterialidades" (LINDÓN, HIERNAUX， 2012: 15).

Este viraje da cuenta de la necesidad de poner la lupa en los sujetos y su accionar espacial, es decir en sus prácticas, pero también en las formas en las que ellos perciben, imaginan y vivencian el territorio, hasta en la dimensión más performativa del espacio (THRIFT, 1996).

En nuestra experiencia de campo referiremos a sujetos que desarrollan prácticas peregrinas en torno a la peregrinación juvenil a pie a la Basílica de Luján. No profundizaremos en la discusión si son peregrinos, devotos, turistas, viajeros, pos-peregrinos, etc. ya que el eje de discusión de esta propuesta es la de ver el rol que ejerce la(s) espacialidad(es) en el fenómeno peregrino poniendo la mirada en los "actuantes" (DI MEO, BULEON, 2005: 29).

Según la Organización Mundial de Turismo (OMT), alrededor del 20\% de los turistas que se desplazan en el mundo lo hacen impulsados por una motivación religiosa. Es decir que más de 300 millones de personas se trasladan anualmente a visitar monumentos y santuarios, o a participar de distintas ceremonias con un código de consumo que escapa, en principio, a los cánones del turismo tradicional (GONZÁLEZ, 2011). En Latinoamérica, países como Brasil tienen una estimación de 20 millones de turistas en este estrato; en México se registra otro tanto, ya que de una población de más de 112 millones de personas, el 10\% se desplaza dentro del país para participar de distintas actividades religiosas, siendo la Virgen de Guadalupe en el Distrito Federal de México, el nodo religioso más importante del país y el quinto en el nivel mundial.

Para la Encuesta de Viajes y Turismo de los Hogares (EVyTH) llevada a cabo en el 2013 por la Subsecretaría de Desarrollo Turístico del Ministerio de Turismo de la Argentina, hubo alrededor de 190.000 desplazamientos por motivos religiosos, concentrados mayoritariamente entre los meses de diciembre y enero. La distribución territorial de estos desplazamientos, según el mismo sondeo, evidencia una concentración en torno al norte (66\%), litoral (13,5\%) y la región central $(8,8 \%)$.

Las cifras solo son descripciones generales que dibujan un panorama muy amplio y lleno de 
huecos con respecto a este tipo de movilidades, sobre todo porque las metodologías utilizadas para acceder a las prácticas no siempre reflejan las formas intersticiales y heterodoxas que se advierten sobre el territorio. Ejemplo de ello es que, según las fuentes periodísticas (basadas en registros de la Policía Federal y las de las autoridades eclesiásticas), para el 5 de octubre del 2013 participaron solamente de la peregrinación juvenil a Luján, entre 2 y 2,5 millones de personas, en el marco de la primera que se desarrollaba bajo el mandato de Francisco I en el Papado, cifra muy superior a lo que muestra la encuesta aludida.

Independientemente de las cifras, sabemos que el viaje religioso es un fenómeno espacial que debe ser analizado poniendo la atención en estos sujetos y las formas en que despliegan las prácticas espaciales, el lugar que ocupan las representaciones y las distintas maneras de narrar la experiencia peregrina.

La elección de poner la mirada en los actuantes del territorio nos obliga -también- a alistarnos en una metodología cualitativa que nos permita adentrarnos en el universo de la subjetividad, y desde allí trazar puentes que den cuenta de las singularidades y generalidades en las que se despliega este fenómeno. Esta perspectiva se entronca con varios aportes de distintos geógrafos que han puesto el acento en la subjetividad y cuya idea más acabada confluye en el denominado constructivismo geográfico.

La geografía constructivista se apoya en la construcción social de los espacios y lugares a partir de la experiencia de los sujetos en acción, por eso

$$
\begin{aligned}
& \text { "un mismo fenómeno, } \\
& \text { una misma realidad, } \\
& \text { puede ser construida } \\
& \text { de diferentes formas } \\
& \text { en función de distintos } \\
& \text { puntos de vista, y de } \\
& \text { acuerdo a las formas } \\
& \text { de nombrarlas, y más } \\
& \text { aún de contarlas” } \\
& \text { (LINDÓN, 20o8: 11). }
\end{aligned}
$$

En el marco de la nueva geografía cultural, la incorporación de estrategias, marcos y categorías provenientes de otras disciplinas sociales permitió resolver el problema de acceder a las vivencias de los otros en los lugares y los espacios, o lo que Yi $\mathrm{Fu}$ Tuan refirió como la experiencia espacial6 $^{6}$ (TUAN, 1977). El autor enfatiza la idea que el espacio tiene una dimensión simbólica que no es independiente de lo sensorial.

Por ello, el acercarse a esas experiencias subjetivas implica un reto metodológico sumamente complejo y 
provocador para los análisis territoriales. En este sentido, dos son las estrategias que hemos sumado para desplegar tal desafío: el desarrollo de un trabajo de campo experiencial y el uso de narrativas espaciales de vida.

En el primer caso se trata de la redefinición de una herramienta que en los estudios geográficos tiene mucha presencia y antigüedad, pero que sin embargo se lo ha concebido desde posturas exocéntricas. El término de trabajo de campo experiencial es acuñado, hacia la década de 1970, por el geógrafo Graham D. Rowles quien propone la recurrencia a campo para lograr el conocimiento interpersonal,

"refiriéndose al
camino intermedio
entre el conocimiento
objetivo, abstracto,
distante y por lo
mismo reduccionista,
y por otro lado, la
búsqueda inacabada
del conocimiento de la
subjetividad espacial
misma, que -en
estricto sentido- es
inaccesible porque
nunca podremos ser el
otro"(LINDóN,
20o8).

La recurrencia a campo se hace obligatoria en distintas circunstancias y situaciones en pos de lograr la empatía necesaria para acercarse a esa "otredad" a través de una observación densa y participante que permita captar el "hacer de los sujetos en el espacio" (LINDÓN, 2008: 15).

El segundo caso colabora con el acceso al universo de la subjetividad y experiencia espacial mediante la oralidad. Se trata del conjunto de los relatos, los discursos, las narrativas, las historia de vida, que son

$$
\begin{aligned}
& \text { "estrategias } \\
& \text { metodológicas } \\
& \text { adecuadas a las } \\
& \text { aproximaciones } \\
& \text { constructivistas para } \\
& \text { encontrar las lógicas } \\
& \text { de la acción (DI MEO, } \\
& \text { BULEÓN, 2005: 37). }
\end{aligned}
$$

Dentro del conjunto de dispositivos de oralidad, nos interesan especialmente las narrativas espaciales de vida, en la medida en qué se trata de una narratividad que pone al espacio en el centro del relato, a partir de una reelaboración subjetiva de los hechos $\mathrm{y}$ procesos. Siguiendo a Lindón (2008), se trata de

$$
\begin{array}{lr}
\text { "un } & \text { relato organizado } \\
\text { y } & \text { secuencializado }
\end{array}
$$


espacio-

temporalmente de

experiencias vividas

por el sujeto en ciertos

lugares. Es una

narración en la cual el lugar -con toda su singularidad- se hace parte de la experiencia allí vivida, influye de alguna forma en la experiencia y le imprime una marca" (LINDÓN, 2008: 19).

No se trata de un recuento de hechos y circunstancias tal cual ocurrieron, sino del resultado de un proceso de reelaboración intersubjetiva que "produce una reconfiguración coherente del mundo" (ROUX, 1999: 37). Ahora bien, es importante sumar como menciona Piña (1999) que

“el relato
autobiográfico no se
limita a ser una suma
de episodios, sino que
en su totalidad se trata
de una articulación
con un sentido
general. Desde el
presente, el pasado
pierde ese estatuto de
simultaneidad
desconcertadora y cuando aún no era pasado, y se convierte en algo inteligible, su sentido brota como evidente, la actualidad lo ordena, tornándolo tolerable y útil” (PIÑA 1999: 4).

Este será entonces nuestro dique de contención, nuestro marco de referencia para interpretar las formas espaciales y transformaciones que han experimentado las prácticas territoriales de los "jóvenes" que caminan anualmente hacia la ciudad de Luján.

\section{La peregrinación juvenil a pie a Luján}

La peregrinación juvenil a pie es una de las más importantes dentro del calendario de peregrinaciones que se desarrollan a la hierópolis de Luján7, tanto por la convocatoria de fieles que congrega como por el despliegue e impacto territorial que produce. Esta significatividad se da tanto en el plano de la subjetividad de la experiencia individual como en su dimensión colectiva.

difusa que tuvo

"Such movement also contains, in various mixtures, the potent ESPAÇO E CULTURA, UERJ, RJ, N. 37, P.116-136, JAN./JUN. DE 2015 combination

of http://www.e-publicacoes.uerj.br/index.php/espacoecultura/ 


individual and
collective experience,
lending it the
possibility of
powerfully shaping
one, the other, or the
relation between the
two" (TAYLOR, 2012:
222).

Su primera edición se llevó a cabo en el 25 de octubre de 1975 cuando alrededor de unos 25.000 sujetos participaron del recorrido desde Liniers al Santuario de Luján. Es interesante destacar que esta práctica se inicia marcada por un contexto posconciliar y de efervescencia de un catolicismo con una fuerte impronta popular, a tal punto que en la organización de la marcha como en la concurrencia de fieles tuvieron una presencia muy fuerte los movimientos de la Pastoral Popular y los Sacerdotes del Tercer Mundo. Uno de los organizadores pioneros recuerda en su narrativa:

algunos olían a
comunismo

("jóvenes", "en la calle" con "los curas villeros" y el "pueblo" que "andan con la Biblia

Latinoamericana”. Me acuerdo que una tarde fui al programa, del Canal Once, de la Tía Valentina. Ni me recibieron. Dejé una gacetilla y me fui a ver el programa a la parroquia. Salió. ¿Qué salió? Solo yo me dí cuenta. Hizo un comentario: con voz venenosa que decía “¿Latinoamérica?” "¿Por qué?” "dejémonos de tanto Latinoamérica!"5

Un antecedente había sido la peregrinación villera desarrollada en el año 1969 y luego continuada por una década, a cargo del equipo Pastoral para las Villas de Emergencia de la Capital Federal. Las mismas se llevaron a cabo en ómnibus "que llegaron a ser más de 130" (VERNAZA, 1989: 24) ${ }^{8}$.

La figura clave en la historia de la peregrinación juvenil a pie es la del sacerdote Rafael Tello, filósofo $\mathrm{y}$ docente universitario de teología, quien había tenido contacto y había participado de las experiencias pastorales de los curas villeros, y de alguna manera había organizado la Pastoral Popular9. De su prédica en distintos ámbitos eclesiásticos surgió la idea de lograr una gran convocatoria a caminar hacia la Basílica de Luján. En 
una reunión con otros líderes religiosos, el propio Tello cristalizó su propuesta en palabras al mencionar:

la juventud puede ir caminando a Luján y convocar a todo el pueblo a que la acompañe [...] Yo los voy a seguir muy de lejos y despacito ${ }^{10}$.

Esa idea pionera -en principio sumamente resistida- fue el disparador de un movimiento que se iría consolidando a lo largo de las décadas siguientes bajo la forma del Movimiento Juvenil Evangelizador.

Los testimonios de los participantes de esa primera edición dan cuenta del carácter heterogéneo que tenía esta primera peregrinación. Recuerda uno de los líderes que:

aunque las motivaciones fueran muchas y distintas, estábamos caminando todos juntos, o como dirían más tarde, estábamos todos mezclados: los de los barrios, los colegios, las parroquias, los curas y las monjitas, los que militaban políticamente, los que fueron a ver qué pasaba, y tantos otros; todos convocados por la misma Madre [...] La realidad era que los jóvenes nos decidíamos a caminar, a ponernos en marcha $^{11}$.

Cabe destacar entonces el componente fuertemente político e ideológico que emergía en esta primera marcha, ya sea desde la organización y pertenencia de sus convocantes hasta el conjunto de símbolos presentes en la misma, sumado al contexto sociopolítico que experimentaba la Argentina a un año de que se llevará a cabo el golpe cívico-militar de 1976. Por ejemplo, el lema de ese año fue "La Juventud peregrina a Luján por la Patria" haciendo explicita mención al contexto político del momento; otro dato es que la marcha estuvo encabezada -como hasta la actualidadpor la imagen de la Virgen de Luján ornamentada con banderas latinoamericanas y flores, y la canción central fue "Tiempo de América" con una clara alusión al contexto sociopolítico de ebullición que experimentaban estos lugares y estas sociedades latinoamericanas.

Las décadas de los ochenta y noventa consolidaron el fenómeno peregrino y las masas de juventudes - 
mayoritariamente parroquiales- se sumaron a la tradicional peregrinación anual a Luján. Las formas del peregrinar se situaban en torno a la práctica colectiva (grupos de pertenencia como los grupos de jóvenes de las parroquias, las juventudes de Acción Católica y las distintas ramas de scouts) y algunos menos que lo hacían en forma desinstitucionalizada (amigos, familiares, etc.). Con rezos, tiempos de silencio y oración y algunas canciones relativas a la liturgia católica se completaba el trayecto de Liniers a Luján, aunque algunos se suman en distintos puntos del camino sagrado. Entonces,

"los peregrinos, en los momentos de solemnidad, al momento del rezo del rosario, predisponen su cuerpo, sus movimientos, $\quad \mathrm{su}$ mirada, su tonalidad, sus gestualidades, preparando de alguna manera los elementos físicos $\mathrm{y}$ hasta la emoción para un compromiso con el rezo" (VERGARA, 2012: 3). Espacialmente, el camino de la peregrinación es ante todo un camino sagrado, reglado por prácticas territoriales que se corresponden con ese universo de lo sagrado y con una identificable separación de una espacialidad profana por fuera del flujo peregrino. 
Imágenes de la primera peregrinación a pie a Luján.
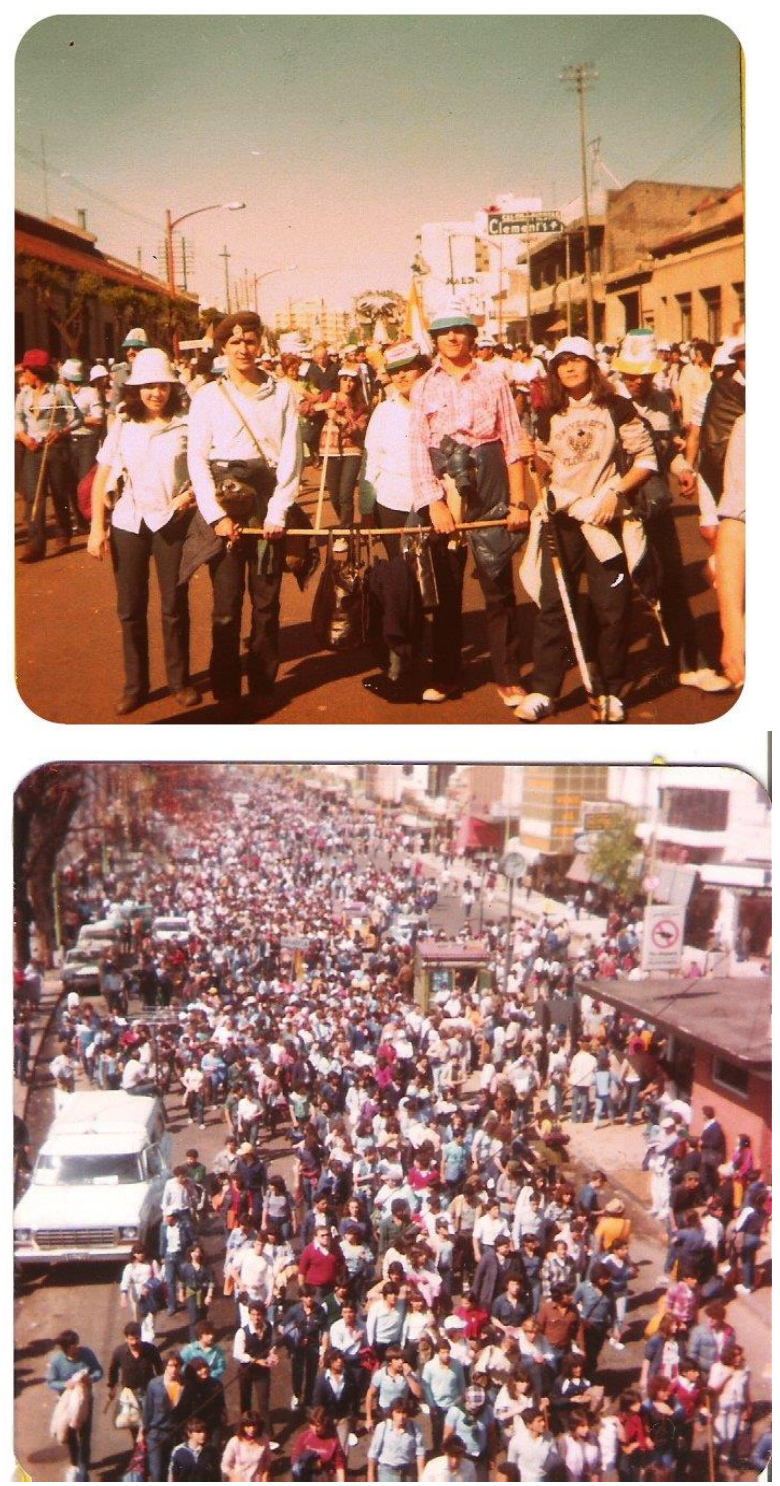

Fuente: http://www.cienciayfe.com.ar.

\section{Cambios en la espacialidad peregrina}

Con el correr de los años el fenómeno de la práctica peregrina se fue consolidando y ampliando. Desde aquel 1975 se llevó a cabo con continuidad todos los primeros fin de semana del mes de octubre, convocando a cientos de miles de fieles que recorren los casi $60 \mathrm{~km}$. a pie en un promedio de diez horas. 
certo modo, com o meio. Um meio que

serve ao ato do sacrifício. Inscreve, portanto, fatos humanos e sociais no espaço, mesmo que de modo efêmero. Em outras palavras, a vida tem pulsão no instante da peregrinação" (NOVO, 2014: 7).
Como podemos ver en el plano, el trayecto se despliega por la ruta 7 (Avenida Rivadavia,) y a lo largo del mismo se instalan una serie de puestos de asistencia (alrededor de un centenar), y también de venta ambulante de todo tipo (alimentos, bebidas, bastones, souvenirs religiosos, etc.). El fenómeno en términos de escenario espacial produce una serie de modificaciones territoriales que afectan a los lugares por donde se peregrina gestando espacialidades superpuestas, dinámicas y complejas. 
Plano de la espacialidad peregrina (Liniers-Luján)

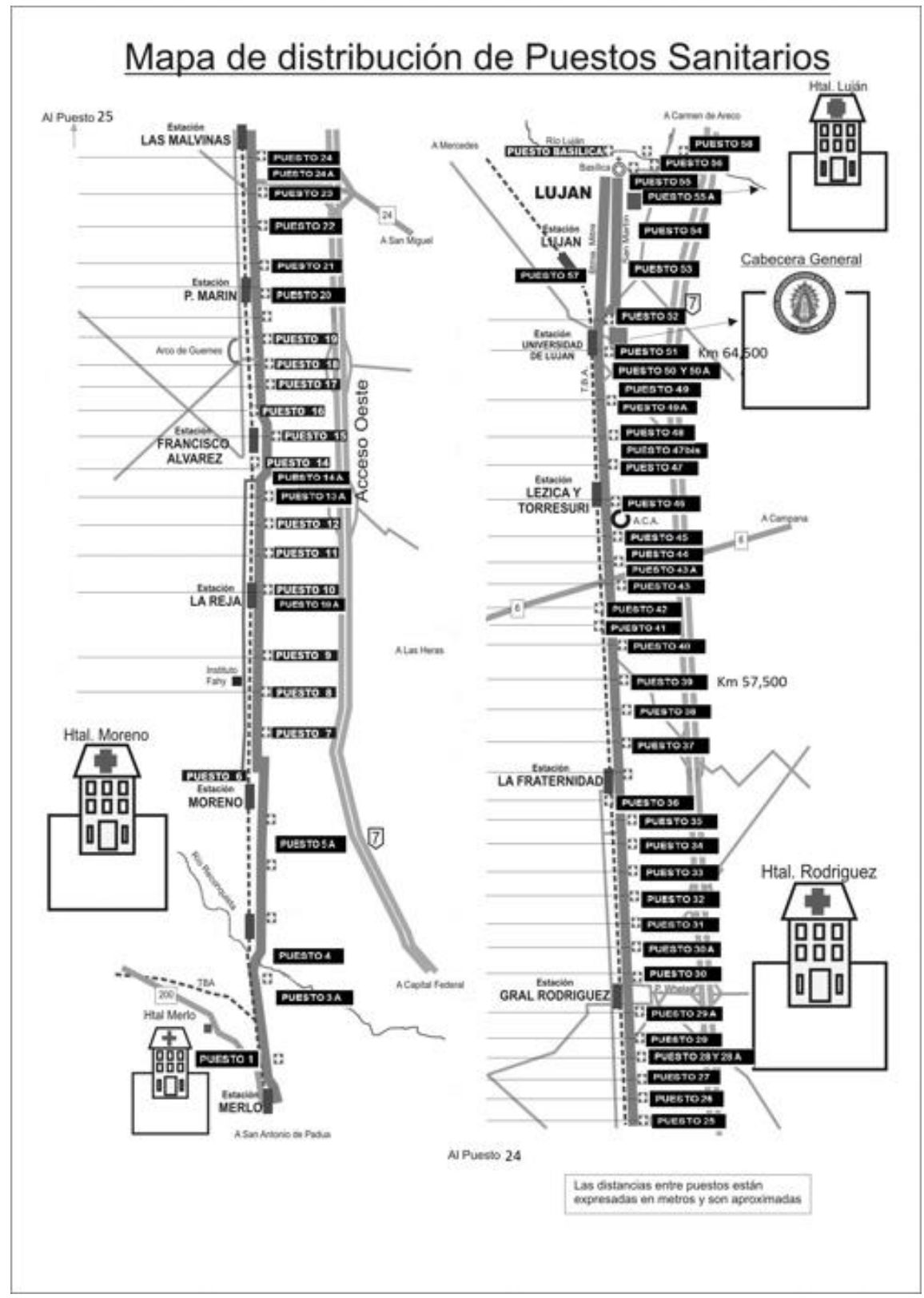

Fuente: www.peregrinos.org.ar (última consulta octubre de 2015).

En las últimas décadas se han ido cimentando una serie de transformaciones en el perfil de los peregrinos, en sus prácticas y representaciones espaciales, y en los aspectos organizativos de la marcha que se materializan en la emergencia de nuevas formas del peregrinar.

La geografía cultural de las religiones ha puesto el énfasis en advertir estos cambios en función de la dualidad prácticas turísticas/prácticas 
peregrinas. Sin embargo, de nuestra perspectiva, el problemas es más complejo y las posibilidades muchas más amplias. No se limita a pensarlo en esa clave dialéctica, sino desde la multiplicidad y complejidad. $^{12}$ De hecho, son las prácticas y las representaciones de los sujetos las que exteriorizan las formas peregrinas y las que definen la sacralidad de los lugares, en la medida en que: "la multiplicidad de prácticas, motivaciones $\mathrm{y}$ singularidades que revelan los viajeros que visitan las hierópolis en el marco de la emergente actividad turísticoreligiosa, vuelve la situación mucho más enmarañada y desdibuja las fronteras entre posibles categorías cerradas" (FLORES, 2011: 84).

Uno de los caminos posibles, y siguiendo la recomendación de Llurdés Coit (1995) consiste en abandonar la supuesta dualidad entre turismo como actividad frívola, trivial y superficial, frente a un aparente carácter genuino, auténtico, sufrido y serio, que se le otorga a la peregrinación. Así un peregrino que desarrolla su práctica religiosa en el espacio, despliega sus ritos, vive la espiritualidad del lugar, puede mutar en un eventual turista que consume bienes y/o lugares turísticos (FLORES, 2011).

Pero además de este cambio, se han generado una serie de transformaciones en las prácticas peregrinas mismas que se vinculan con el surgimiento de formas pos-modernas del peregrinar (ROSENDAHL, 2006). $\mathrm{Al}$ respecto, la geógrafa brasileña señala que:

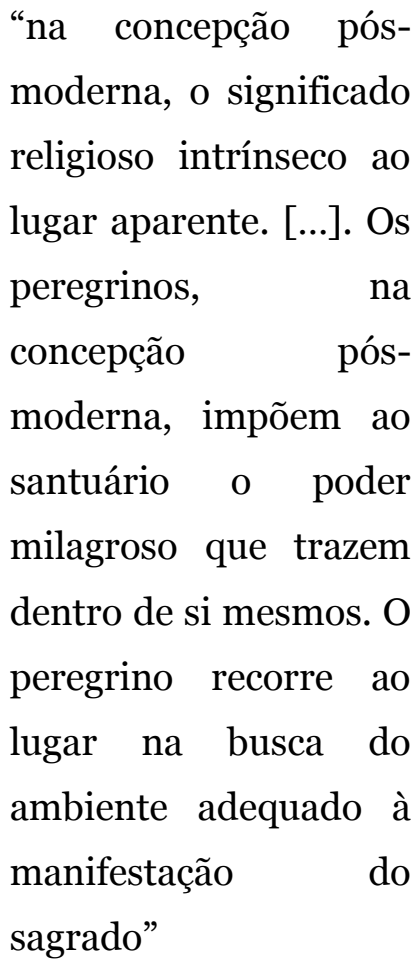

(ROSENDAHL, 2006: 34).

Se genera una ruptura del tradicional modelo de espacios/prácticas sagrada(os) y espacios/prácticas profanas; esta distinción que delimitaba formas de comportarse y actuar en el (y con el) espacio parece ser mucho menos evidente $\mathrm{y}$ tajante en los nuevos peregrinos. Algunas de estas expresiones podemos advertirlas en las formas actuales que adopta la peregrinación juvenil a pie a Luján y que dan cuenta de la flexibilidad de las 
lógicas peregrinas que Rosendalh (2006) categoriza como pos-modernas.

En estas territorialidades -como adelantábamos- se evidencia una clara ruptura entre la distinción de espacios profanos y sagrados; no por el hecho de su existencia en sí, sino más bien por las prácticas y las representaciones que los sujetos construyen sobre esto. No hay prácticas sagradas y otras profanas; lo mismo con su locus. Al rezo, al silencio y los cánticos litúrgicos se le pueden sumar otras acciones como escuchar y cantar canciones seculares (cumbia, rock, por ejemplo) o consumir alcohol y fumar tabaco y/o marihuana, sin que esto constituya un conflicto ni altere el paisaje peregrino.

Durante el recorrido, el rezo del rosario de los grupos parroquiales se mezcla con la música de cumbia que escuchan otros peregrinos en forma simultánea, generando una yuxtaposición entre prácticas sagradas $\mathrm{y}$ otras profanas. Inclusive, se suelen dar procesos de apropiación y mixtura que hacen del fenómeno algo más complejo aún. Vergara (2012) advierte en este sentido-

“cómo muchos
peregrinos,
pertenecientes

distintas parroquias, pasean la imagen de la Virgen en carritos desde los cuales se escucha música popular, proyectando así en un mismo espacio, una imagen que pertenece al ámbito de lo sagrado y una música que pertenece al ámbito de lo profano"

(VERGARA, 2012: 5).

De manera similar ocurre con la espacialidad y los lugares. Ya no hay una sacralidad propia de ciertos espacios y una profanidad propio de otros. Se puede descansar dentro de la Basílica, comer o acostarse a dormir, aunque no sean prácticas espaciales propias de éstos lugares. Son entonces los sujetos peregrinos los que activan la sacralidad/profanidad en determinados momentos a través de sus acciones en el territorio, dotando de estas cualidades al espacio en el que se enmarcan.

Otra característica que fue tomando la peregrinación en los últimos tiempos es un proceso de desinstitucionalización e individuación del peregrinar. Año a año se fueron reduciendo los grupos parroquiales organizados para dejar paso a los peregrinos cuentapropistas que advierten en la peregrinación no necesariamente una motivación exclusivamente religiosa, sino también un ámbito de sociabilidad e inclusión comunitaria. El componente político que identificaba a las primeras peregrinaciones no desapareció, pero sí 
Peregrinos descansando en el interior de la Basílica.

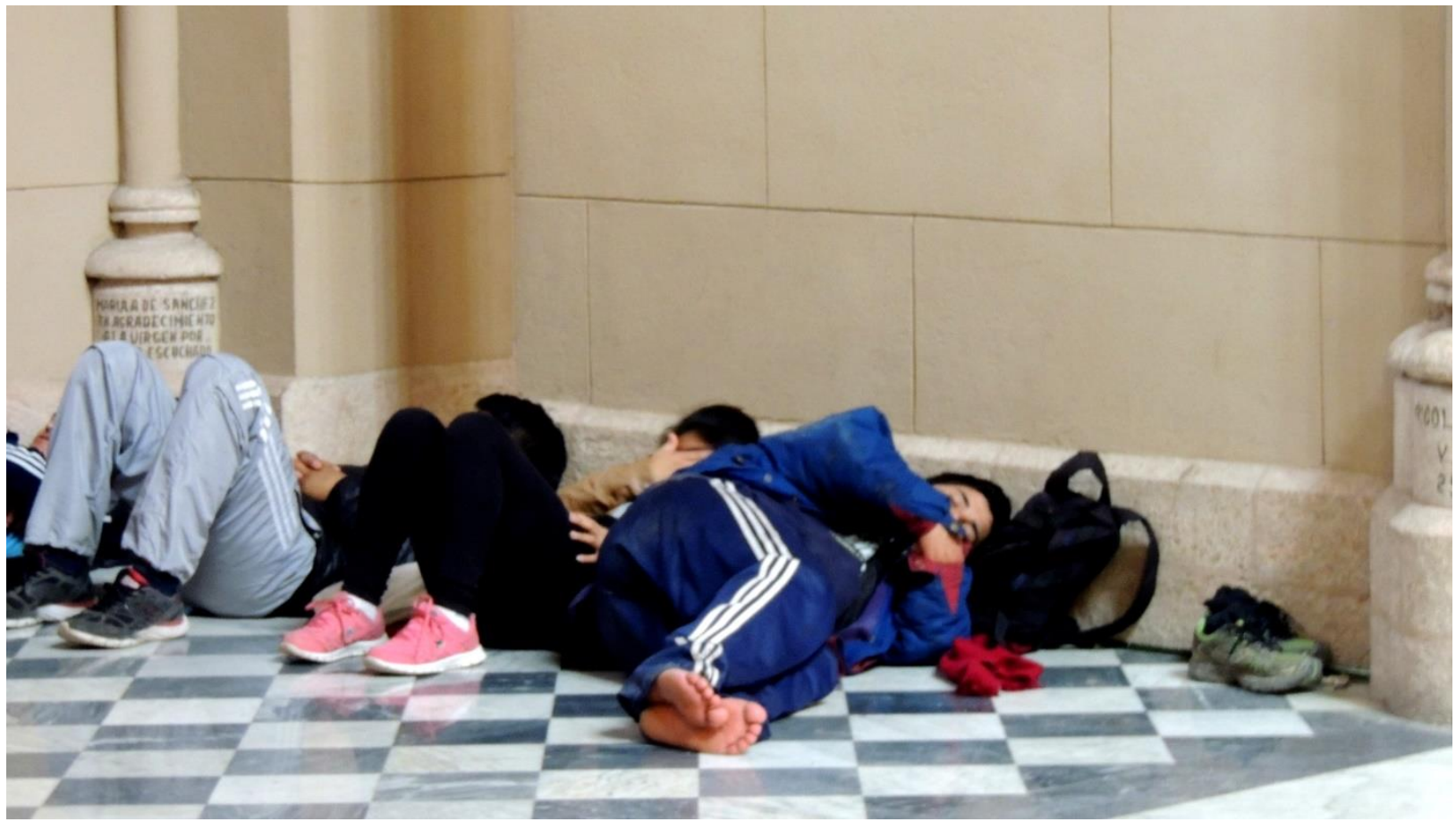

Fuente: fotografia propia, 2015.

Finalmente, cabe preguntarse acerca de dos cuestiones que caracterizan particularmente a la peregrinación juvenil a pie a Luján: por un lado, la idea de juventud(es) que se vislumbra en los sujetos peregrinos, $\mathrm{y}$ por otro lado, el matiz de esta práctica espacial como un fenómeno de religiosidad popular, con todas las complejidades que implica esta categoría.

$\mathrm{Al}$ respecto del primer aspecto es visible que desde el origen de la peregrinación de 1975 estuvo teñida fuertemente por la presencia de la juventud (en términos etarios exclusivamente), tanto en los participantes como en las comisiones organizadoras. También los discursos, los lemas, los cánticos y la morfología general de la marcha estuvieron direccionados a los jóvenes específicamente. De hecho,

"en los años setenta y ochenta, la prédica y el mensaje que los jóvenes compartían al terminar la misa era la clave de la Pastoral Juvenil anual de la región de Buenos Aires. [...] En la actualidad el trabajo está orientado a crear gestos que 
identifiquen el sentir de todo el pueblo al participar de las celebraciones en la plaza" (GALLI, et. al., 2004: 183).

Este giro en los discursos y los destinatarios se dio - también- en un contexto de modificación del perfil de los asistentes y de las subjetividades respecto a los imaginarios sobre la juventud. Es que en este sentido, la idea de juventud no está relacionada exclusivamente con una cuestión etaria, sino más bien que se trata de modalidades de ser joven que dependen de la edad, la generación, el crédito vital, la clase social, el marco institucional y el género (MARGULIS, URRSTI, 1996) ${ }^{14}$.

En segundo término, la presencia de un catolicismo popular se manifiesta desde el origen de la misma, con ciertos matices que fueron haciendo que se diluyera ese componente y que se volviera mucho más heterogéneo y

Notas

Investigador Adjunto de Carrera del CONICET. Docente auxiliar e investigador del Departamento de Ciencias Sociales de la Universidad Nacional de Luján.

2 Para el caso particular de la geografía, recién hacia la década de 1980 la movilidad religiosa se presenta como un tema posible de ser abordado en clave territorial. Sin embargo, muchas de estos trabajos se sostienen en el viejo paradigma que define a la peregrinación solamente como una especie de viaje religioso (COLLINS-KREINER, 2010).

3 "O conceito de imaginação geográfica firma-se nas proposições teóricas que almejam dar relevo à interpretação dos fenômenos sócio-espaciais quer estejam associados a uma perspectiva da natureza, quer estejam atrelados às práticas simbólicas cujos elementos discursivos indicam um processo que envolve a produção de paisagens culturais, de lugares versátil. No obstante creemos que es un marco viable pensar a la espacialidad peregrina como una manifestación de religiosidad popular pero entendida "más que como una espiritualidad paralela, como una religiosidad trasversal a la institución" (DE LA TORRE, 2012: 511). La presencia de nuevas formas de mediación con el universo de lo sagrado y de mixturas y sincretismos que año a año superan la imaginación religiosa de los fieles, delinean estos nuevos paisajes peregrinos, estas nuevas rutas sagradas que se reconfiguran y revientan las espacialidades históricamente construidas.

El desafío como geógrafos cualitativos es utilizar todo el arsenal de herramientas posibles que nos permitan acercarnos a leer esa complejidad del territorio religioso, sin perder la mirada del científico, pero estando atentos a captar este universo de subjetividades que construyen estos paisajes y le dan razón de ser.

ou territórios e territorialidades simbólicas pelas quais uma construção identitária é sempre presente. Tornase possível, portanto, e colocamos aqui a proposta para este texto, o entendimento das representações do sagrado contextualizando na seara da imaginação geográfica” (COSTA, 2012: 49).

4 Para el caso argentino se destaca el trabajo de la Dra. Cristina Carballo sobre la peregrinación de los gauchos a la Basílica de Luján. Véase: Carballo Cristina (2012), "La corporalidad como nuevos territorios de espacialidad religiosa" en Espaço e Cultura, 32, JulhoDezembro, Río de Janeiro.

5 "Experiencia es un término que abarca las diferentes maneras a través de las cuales una persona conoce y construye la realidad. Estas maneras varían desde los sentidos más directos y pasivos como el olfato, paladar y tacto, hasta la percepción visual activa y la manera indirecta de simbolización [...]. Los espacios del 
hombre reflejan la calidad de sus sentidos y su mentalidad. La mente frecuentemente extrapola más allá de la evidencia sensorial”. (TUAN, 1983: 9-18).

${ }^{6}$ La ciudad de Luján está ubicada en el oeste de la provincia de Buenos Aires a unos $70 \mathrm{~km}$. de la ciudad de Buenos Aires. La producción social del territorio de Luján se ha cimentado sobre la idea de una dimensión religiosa del territorio originada a partir de una mariofanía ocurrida en el siglo XVIII.

Actualmente es el principal centro de peregrinación y turismo religioso de la Argentina que recibe anualmente entre 3 y 5 millones de visitantes.

7 Narrativa de Ricardo Larken en: http://parroquiasanbenito.com.ar/pre/2009/10/la1\%C2\%BA-peregrinacion-juvenil-a-lujan-25-deoctubre-de-1975/ (última consulta junio de 2015).

8 "En el volante que convocaba a la peregrinación se expresaba: 'Virgen de Luján, ayúdanos a transformar nuestras villas miseria en barrios obreros' y se pedía especialmente por tres intenciones: 'por un techo digno para nuestros hijos; por un salario justo y un trabajo humano; por una patria sin miseria ni explotación' (TOURIS, 2013: 74).

9 "Concluido el Concilio Vaticano II, comienza a cristalizarse en la Argentina una corriente teológica que proponía pensar la relación entre Dios y la historia. Se trataba de un grupo que crecía impulsado por un anhelo común en Latinoamérica: la liberación de los pueblos. [...] Si bien la acentuación seguía estando sobre el pueblo, la puerta de acceso no sería la liberación, sino la cultura” (ALBADO, 2013: 220).

${ }^{10}$ Narrativa de M. extraído de Galli et. al. (2004).

${ }^{11}$ Narrativa de M. extraído de Galli et. al. (2004).

$12 \mathrm{Al}$ respecto de la esta discusión véase: Flores, F. (2011), ¿̇uristas o peregrinos? Prácticas en torno al fenómeno religioso en San Nicolás de los Arroyos en Revista Transporte y Territorio, $\mathrm{N}^{\circ} 5$, Universidad de Buenos Aires, Diciembre de 2011, pp. 72-88. Disponible en: www.rtt.filo.uba.ar/RTT00505072.pdf. Buenos Aires.

${ }_{13}$ Por ejemplo en la peregrinación del año 2008, en pleno conflicto del gobierno argentino con las patronales rurales, se advirtieron columnas con banderas que decían: "Por la Paz de la Patria y el campo caminamos hacia la Virgen de Luján". En sintonía con este componente político, en 2014 un grupo de peregrinos de la Agrupación política "La Cámpora" participó con una bandera que proclamaba: "Madre caminamos por un pueblo en paz sin buitres" en alusión al conflicto que la Argentina mantenía con los holdouts o "fondos buitre".

14 "La condición juvenil hace referencia a un estado o situación en que se encuentra una individualidad y, en consecuencia, se inscribe en cada uno de los sucesivos modos de ser que no necesariamente están adscritos a una linealidad y menos a una teleología y que, por el contrario, se relacionan con las colaboraciones psíquicas de las experiencias en que haya estado inmersa cada individualidad" (VILLA SEPÚLVEDA, 2011: 151).

\section{Referencias Bibliográficas}

ALBADO, O. (2013), "La Pastoral Popular en el pensamiento del Padre Rafael Tello. Una contribución desde Argentina a la Teología Latinoamericana" en Franciscanum, IV, 160, diciembre de 2013.

CARBALLO, C. (2010), Hierópolis como espacios en construcción: las prácticas peregrinas en la Argentina" en Rosendahl, Z. (Org.) Trilhas do sagrado, Rio de Janeiro: Ed. UERJ.
CARBALLO, C. (2012), "La corporalidad como nuevos territorios de espacialidad religiosa" en Espaço e Cultura, 32, Julho-Dezembro, Río de Janeiro.

COHEN, E. (1992). "Pilgrimage centers, concentric and excentric", Annals of Tourism Research, 19(1), 33-50.

COLLINS-KREINER, N. 2010) "The Geography of Pilgrimage and Tourism: transformations and Implications for Applied Geography”, Applied Geography 30, 1: 153-164.

DE LA TORRE, R. (2012), "La religiosidad popular como 'entre-medio' entre la religiosidad institucional y la espiritualidad individualizada" en Civitas, 12, 3, Porto Alegre.

DI MEO, G. y P. Buleon (2005), L'espace social: lectura géographique des sociétés, Paris: Armand Collin.

FLEISCHER, A. (2000). "The Tourist behind the Pilgrim in the Holy Land". International Journal of Hospitality Management, 19, 311-326.

FLORES, F. (2011), ¿'Turistas o peregrinos? Prácticas en torno al fenómeno religioso en San Nicolás de los Arroyos en Revista Transporte y Territorio, $\mathrm{N}^{\mathrm{O}} 5$, Universidad de Buenos Aires, Diciembre de 2011. Disponible en: www.rtt.filo.uba.ar/RTTo0505072.pdf.

GALLI, M. et. al. (2004), Seguimos caminando... Aproximación socio-histórica, teológica y pastoral de la peregrinación juvenil a Luján, Buenos Aires: Guadalupe.

GONZÁLEZ, M. (2011), "Turismo religioso: una experiencia de fe que crece y se suma al boom de los feriados" en Diario Bae, Buenos Aires, 17 de abril de 2011.

COSTA (2012), O. J. L da "A imaginação geográfica e as representações dos lugares sagrados", en Espaço $e$ Cultura, 32, Julho-Dezembro, Río de Janeiro.

LINDÓN, A. (2008), "De las geografías constructivistas a las narrativas de vida espaciales como metodologías geográficas cualitativas" en Revista da ANPEGE, Vol. 4, Porto Alegre.

LINDÓN A. y Hiernaux D. (dirs.) (2012), Geografías de lo imaginario, Barcelona: Anthropos.

LLURDÉS COIT, J. C. (1995), "Turismo y religión como base de un proyecto turístico. El ejemplo de la Ruta Transpirenaica de peregrinaje LourdesMontserrat" en Cambios regionales a finales del siglo XX, Salamanca, AGE.

MARGULIS, M., URRESTI, M. (1996), La Juventud es más que una palabra, Buenos Aires: Biblos.

NOVO, Cássio Lopes da Cruz (2014), "Desfilar e Peregrinar: pontos de aproximação e convergências entre blocos de rua e peregrinações pós-modernas”, $I$ Coloquio Latinoamericano "Territorios, fiestas y paisajes peregrinos: Cartografias sociales de lo sagrado en el siglo XXI, Quilmes, Luján, 19 y 20 de noviembre de 2014.

OLIVEIRA, Jefferson Rodrigues de (2014), "A prática ritualística de peregrinar na pós-modernidade: os caminhos da fé em Cachoeira Paulista (SP)”, I Coloquio Latinoamericano "Territorios, fiestas y paisajes peregrinos: Cartografias sociales de lo 
sagrado en el siglo XXI, Quilmes, Luján, 19 y 20 de noviembre de 2014.

PIÑA, C. (1999), "Tiempo y memoria. Sobre los artificios del relato autobiográfico", En Proposiciones, 29, México.

PORCAL GONZALO, M. (2006), "Turismo cultural, turismo religioso y peregrinaciones en Navarra. Las javieradas como caso de estudio" en Cuadernos de Turismo, $\mathrm{n}^{\circ} 18$, Universidad de Murcia, Murcia, España.

PORIA, Y., Butler, R., \& Airey, D. (2003), "The core of heritage tourism, distinguishing heritage tourists from tourists in heritage places" Annals of Tourism Research, 30(1).

PORIA, Y., Butler, R., \& Airey, D. (2004), How tourists decide which heritage site to visit, Tourism Review, 59(2), 12-16.

PORIA, Y., Biran, A., \& Reichel, R. (2006). "Tourist perceptions, personal vs. non personal", Journal of Heritage Tourism, 12, 121-132.

ROUX, M. (1999), Géographie et complexité: les espaces de la nostalgie. París: L' Harmattan.

ROSENDAHL, Z. (2006), "Cultura, Turismo e Identidade". En Borzacchiello, J.; Lima L.; Elias, D. (Orgs.), Panorama da Geografia Brasileira, v.1, , São Paulo: Annablume.

Smith, V. (1992), "Introduction. The quest in guest". Annals of Tourism Research, 19(1), 1-17.
SOJA, E. (1996), Third space. Journeys to Los Angeles and Other Real-and-Imagined Places, Malden, Massachussets, Oxford:Blackwell.

TAYLOR, L. (2012), "Pilgrimage, Moral Geography and Contemporary Religion in the West" en Jansen W. y Notermans, C., Gender, Nation and Religion in European Pilgrimage, Ashgate.

THRIFT, N. (1996), Spatial Formations, Londres: Sage.

TUAN, Y. (1977), Space and Place. The perspective of the experience, Minneapolis: University of Minnesota.

TOURIS, C. (2013), "Catolicismo popular e imaginario liberacionista en los años 70. El caso de las peregrinaciones villeras a Luján y del Movimiento Villero Peronista" en Foguelman, P, M. Ceva y C. Touris, El culto mariano en Luján y San Nicolás. Religiosidad e historia regional. Buenos Aires: Biblos.

TUAN, Y. (1983), Espaço e lugar: a perspectiva da experiência. São Paulo: Difel.

VERGARA, S. (2012), "Corporeidades peregrinas: un análisis sobre las corporeidades construidas y proyectadas durante la peregrinación a la Virgen de Luján”, Facultad de Cs. Sociales de la UBA.

VERNAZZA J. (1989), Para comprender una vida con los pobres. Los curas villeros, Buenos Aires: Guadalupe.

VILLA SEPÚlVEDA, M. (2011), "Del concepto de juventud al de juventudes y al de lo juvenil" en Revista Educación y Pedagogía, vol. 23, núm. 60, mayoagosto, 2011

\title{
SPATIALITIES PILGRIMS: THE CASE OF THE YOUTH PILGRIMAGE ON FOOT TO LUJAN CITY.
}

\begin{abstract}
SINCE 1975 IT IS HELD ONE OF THE MOST IMPORTANT CATHOLIC PILGRIMAGE OF ARGENTINA: YOUTH WALKING PILGRIMAGE TO THE BASILICA
\end{abstract} OF LUJÁN. UNDER THE SLOGAN "THE YOUTH PILGRIMAGE TO LUJAN FOR THE HOMELAND", ON OCTOBER 25, 1975 ABOUT 30 THOUSAND DEVOTEES PILGRIMAGE FOR THE FIRST TIME TO THAT ARGENTINA HIERAPOLIS. THE PRESET TOUR CONSISTS OF ABOUT $60 \mathrm{KM}$. APPROXIMATELY FROM THE DISTRICT OF LINIERS IN CABA TO THE BASILICA IN LUJAN, AVERAGING 15 HOURS OF WALKING. NOT ALL OF THEM PERFORM IT COMPLETELY, BUT SOME OF THEM JOIN IN THE VARIOUS LOCATIONS OF THE ROUTE, ESPECIALLY BETWEEN MORON AND MORENO. THROUGHOUT THE JOURNEY AND THE PILGRIM PRACTICE SPATIALITIES PLAY A CENTRAL ROLE IN THE NARRATIVE OF THE DEVOTEES OF A HETEROGENEOUS ORIGIN. THE OBJECTIVES OF THE WORK ARE: ON ONE HAND, ANALYZING USING THE QUALITATIVE METHODOLOGY - 
TIED TO NEW CULTURAL GEOGRAPHY - THE ORIGIN AND TRANSFORMATION OF THE PILGRIM PRACTICE IN GENERAL. ON THE OTHER HAND, EXPLORING THE FORMS THAT THIS PILGRIMAGE ADOPTS IN PARTICULAR "THE YOUTH" IN RELATION TO THE CONTEXT AND THE WIDEST RANGE OF THE CALENDAR PILGRIM CITY OF LUJÁN.

KEY WORDS: PILGRIMAGE - SPACIALITY - RELIGIOUSNESS. 